\title{
Transhorizon VHF Telemetry from Ocean Moorings
}

\author{
DAVID A. BROOKS \\ Texas A\&M University, Department of Oceanography, College Station, TX 77843
}

(Manuscript received 4 November 1983, in final form 1 February 1984)

\begin{abstract}
During long deployment periods, it is desirable to communicate remotely with moored or drifting instruments. In addition to providing access to the data set as it is collected, a telemetry capability provides insurance against undetected instrument failure and the associated risk of jeopardized scientific objectives. As part of an ongoing study in the Gulf of Maine, an experimental VHF transhorizon telemetry system was recently deployed with a current meter mooring. The refractive properties of the marine boundary layer made it possible to transmit data with about $70 \%$ reliability over a $100 \mathrm{~km}$ distance, or about ten times the optical-horizon distance for the antenna heights used. During weather anomalies, large signal-strength enhancements occurred, and these could easily be exploited to increase the data-transfer reliability.
\end{abstract}

\section{Introduction}

Modern oceanography relies on moored and drifting instrumentation. Typically, a deployed instrument has provisions for internal storage of the data set, which remains unavailable until the instrument is recovered. In case of relatively short deployment periods, the post facto availability of data may be tolerable, but for longer deployments and coordinated field programs this uncertainty becomes a matter of serious scientific and economic concern. An undetected, early failure of a critical instrument on a longterm mooring can result in jeopardized scientific objectives and much wasted time.

Redundant instrumentation can be used to lessen the impact of premature failures, but for maximum cost-effectiveness and scientific utility it is desirable to have ready access to the data from deployed instruments. There are, of course, many telemetry schemes of varying complexity, cost and usefulness by which the data transfer can be accomplished. This paper describes some preliminary experiments with a transhorizon VHF telemetry system that is well suited for use in the coastal or continental shelf region. The special appeal of the described system is its low cost, relative simplicity, and widespread applicability to ocean studies in the coastal zone. Its scientific merits include real-time access to data and instrumentwelfare information, the ability to coordinate at-sea ship operations with moored or drifting instrument measurements, and an "early-warning" capability for monitoring and prediction applications.

\section{Fundamentals of VHF propagation}

Very-high-frequency (VHF) radio waves, which by definition have frequencies greater than $30 \mathrm{MHz}$, are not usually reflected by the ionosphere. The "skip" zones which are commonly observed for lower frequencies are generally absent for VHF. Because of this, VHF propagation is commonly thought to be useful only over line-of-sight distances. However, all electromagnetic waves are refracted to some extent as they travel in the lower atmosphere (the troposphere), and this effect becomes more noticeable as frequency increases, resulting in a VHF radio horizon somewhat more distant than the geometric horizon. Under certain tropospheric conditions, the refractivity can become large enough to "trap" a propagating VHF wave in a near-surface layer known as a duct, which has properties similar to a waveguide. Tropospheric ducting can provide strong-signal VHF paths far beyond the geometric horizon.

The atmospheric index of refraction $N$ is a function of temperature, pressure and moisture content, expressed in the form (Bean and Dutton, 1968)

$$
N=\frac{c_{1}}{T}\left(p+c_{2} \frac{q}{T}\right)
$$

where $c_{1}, c_{2}$ are constants, and $T, p$ and $q$ are, respectively, the temperature, pressure and partial pressure of water vapor. In the climatological-mean atmosphere, it is found that the vertical dependence of $N$ approximately follows an exponential law such as

$$
N(z)=N_{s} \exp (-z / H),
$$

where the surface value $N_{s} \approx 330$ and the scale height $H \approx 7 \mathrm{~km}$. Since it is the vertical gradient of $N$ which produces ray curvature, the exponential law has the advantage that the near-surface gradient is directly proportional to the surface refractivity $N_{s}$, i.e., 


$$
\frac{d N}{d z}=-N_{s} H^{-1} \exp (-z / H)
$$

Thus, regions of relatively large $N_{s}$ tend to be well correlated with regions of enhanced refraction, in the long-term mean.

To illustrate, the seasonal variation of $N$ versus height and time of day is shown in Fig. 1 for a semitropical, humid station (Brownsville, TX) and for a higher latitude station more influenced by continental air masses (Portland, ME). These figures are taken from Bean and Dutton (1968), and they are based on 5-year averages from radiosonde data. The generally higher surface values and diurnal range at Brownsville reflect the high summer temperature and resultant high water vapor capacity at the periphery of the Gulf of Mexico. In contrast, the effect of cold, dry continental air is evident in the low winter values of $N$ at Portland. The comparison shows the typical climate-related changes in refractivity from humid, subtropical areas to marine-modified areas in the lee of a continental land mass. Bean and Dutton also show that a vertical gradient of $\approx 150 \mathrm{~N}$ units $\mathrm{km}^{-1}$ is required to support a near-surface VHF duct. Consequently, the climatological mean refraction in the lower atmosphere (Fig. 1) is insufficient to provide a surface duct on a long-term basis, even in tropical climates.

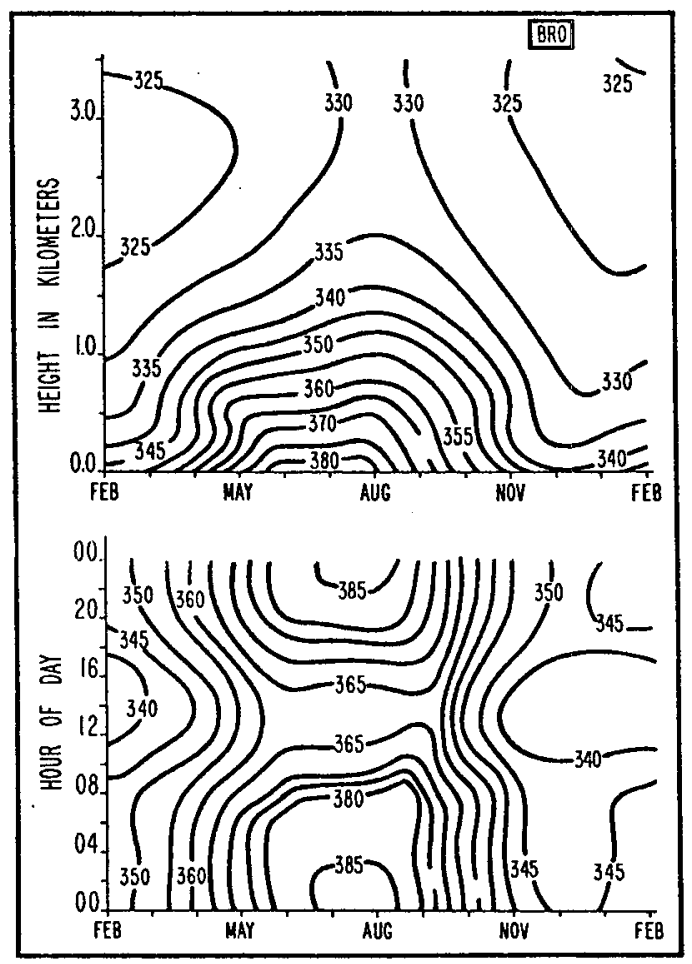

Diurnal, seasonal, and vertical variation of Nofor Browneville, Tex. (BRO).

\section{a. Synoptic weather effects}

On the time scale of day-to-day tropospheric weather perturbations, the $N(z)$ profile can vary markedly from the long-term mean. The principal causes of the variations are abrupt changes in temperature and humidity associated with interacting air masses during the passage of polar frontal disturbances. When cold, dry polar air behind a cold front pushes under the warm, moist tropical air ahead of it, temperature and moisture inversions and sharp gradients in refractivity can occur at the air mass boundary. The vertical gradient of $N$ in such inversions can greatly exceed the critical value for ducting, even though the near-surface gradient may be smaller than it would have been in the absence of the inversion.

Super-refractive propagation can be maintained in tropospheric ducts for hundreds or even thousands of kilometers by a ray-bending mechanism similar to that which supports acoustic wave propagation over long distances in the oceanic SOFAR channel. Duct formation is also caused by nocturnal radiative cooling of the lower troposphere, which introduces a diurnal modulation of the process. In coastal marine climates, land and sea breezes may produce temperature-inversion ducts, and moisture gradients over the ocean contribute to downward refraction and ducting in the

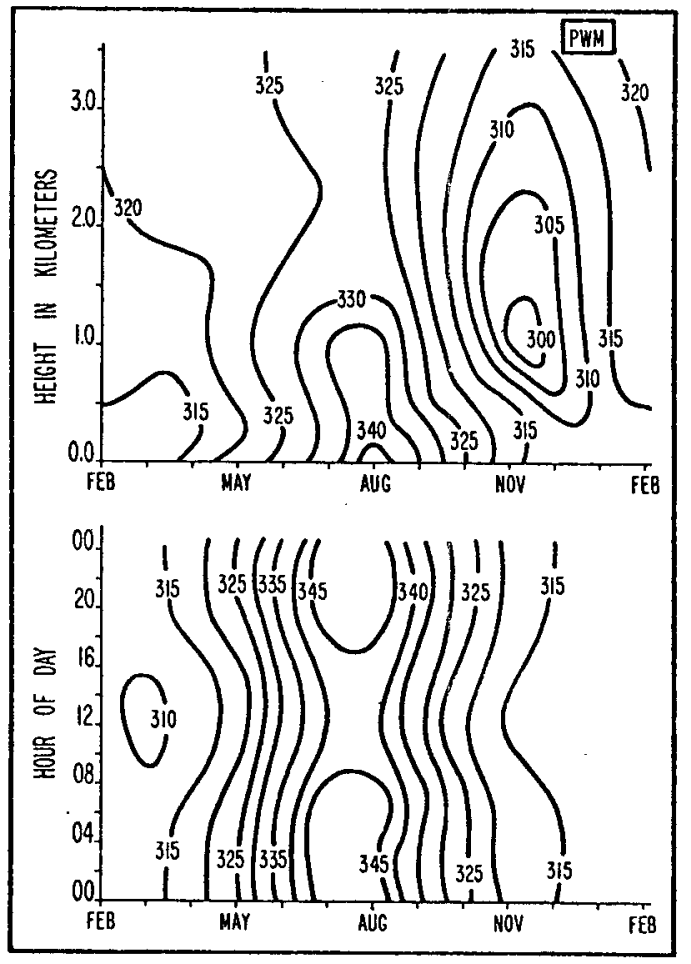

Diurnal, seasonal, and vertical variation of $\mathrm{N}_{0}$ for Portland, Me. (PWM).

FIG. 1. Contours of mean refractive index as a function of height and season for Brownsville, TX (BRO) and Portland, ME (PWM). (From Bean and Dutton, 1968.) 
marine boundary layer. All of these mechanisms are most effective in stably-stratified atmospheres, in which the layered structure perseveres against the disorganizing influence of turbulence.

- Since frontal weather disturbances usually move rapidly from west to east across the United States, the VHF propagation "openings" associated with frontal inversions are transient phenomena swept along with the front. An example of the local refractivity variations associated with a cold front is shown in Fig. 2. The data in this vertical section, also from Bean and Dutton (1968), are contoured versus time in so-called $A$-units, which emphasize the departure of the observed $N(z)$ profile from the mean exponential profile, i.e.,

$$
A(z, t)=N(z)+N_{s}[1-\exp (-z / H)]
$$

Thus, if the observed $N(z)$ profile happened to coincide with the exponential mean profile, the resultant $A(z)$ profile would be a straight vertical line with value $N_{s}$.

The example shows sharp gradients in $A(z, t)$ associated with the passage of a cold front over Lake Charles, Louisiana. The effects of the temperature inversion between the inflowing tropical air ahead of the front and the cold air behind it are seen in the closely-spaced contours near the leading edge of the front at a height of $\sim 750 \mathrm{~m}$. Strong VHF refraction would likely be associated with the feature, but its duration at any given location would be limited to an hour or less. The region of small vertical gradients behind the front is associated with the thick layer of cold, dry continental air, which has refractive prop- erties similar to the mean exponential profile. While the example shown is for a mostly tropical region, refractive disturbances of similar or even larger magnitude, relative to the climatological mean situation, can be expected at higher latitude locations in the continental lee, where air mass contrasts are very large during frontal passages. Thus, weather-related VHF propagation anomalies can be very significant at northern stations such as Portland, Maine, even though the mean atmosphere may be less refractive there than in more tropical latitudes (e.g., see Fig. 1).

\section{b. Oceanic influences}

The moisture flux from the ocean to the atmosphere is essentially confined to the thickness of the atmospheric boundary layer, which may range from a few hundred meters to several kilometers (to the tropopause). The moisture content of the air generally decreases with height in the boundary layer, producing a VHF refractive tendency which is additive to the weather anomalies just mentioned. The effectiveness of the addition depends on the details of the boundary layer. Cold, dry, continental air moving offshore over a warm ocean produces the most intense evaporative moisture fluxes. When this situation is accompanied by a stable atmospheric boundary layer, vertical turbulent mixing is suppressed, which favors the formation of a refractive surface duct. Conversely and less frequently, warm moist air flowing over cold water can provide favorable propagation if fog is produced in the surface boundary layer. In such cases,

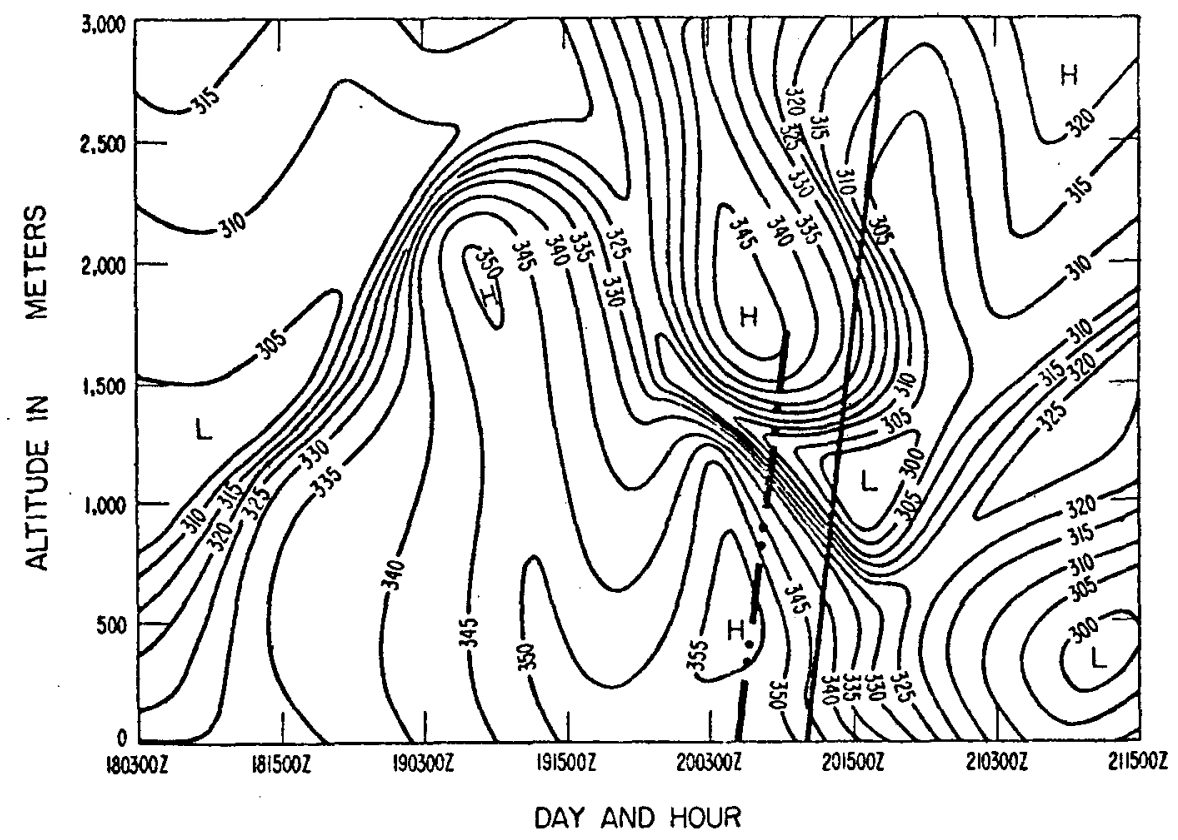

FIG. 2. Vertical section of refractive index in $A$-units (see text), contoured as a function of time for Lake Charles, LA. (From Bean and Dutton, 1968.) 
the lower atmosphere is completely saturated, while the air above may be considerably dryer, producing sharp gradients of moisture content and potentially strong refraction.

In 1947, a surface duct in the marine boundary layer over the ocean off New Zealand was carefully mapped by an aircraft and ship survey (Project Canterbury; Unwin, 1951). Model calculations based on the Canterbury data showed super-refractive conditions in the lower few hundred meters of the marine boundary layer. The effect extended to at least 200 $\mathrm{km}$ offshore, and an abrupt return to non-ducting conditions occurred only a few kilometers inland from the ocean. The important point is that, over the ocean surface, refractive VHF propagation may be useful for transhorizon telemetry even in the absence of synoptic weather disturbances such as cold fronts.

\section{A pilot experiment in the Gulf of Maine}

As part of an ongoing study of the circulation in the Gulf of Maine, two instrument moorings were deployed for the summer months of 1983 (Fig. 3). Mooring A was located over the eastern edge of Wilkinson Basin, in water $200 \mathrm{~m}$ deep, about 100 km south of Boothbay Harbor, Maine. An experimental VHF telemetry system was included on a surface buoy attached to mooring $A$, which supported Aanderaa model RCM-4 current meters at depths of 30 and $180 \mathrm{~m}$. The instruments recorded current speed and direction, water temperature, and water conductivity (related to salinity), and this information was also sent by acoustical means to the surface telemetry buoy. The data were then transmitted to a base station located at Boothbay Harbor.

The experiment demonstrated the usefulness of VHF telemetry. Some unexpected current speeds and directions were noted, providing real-time information which would have been very important in an experiment with coordinated ship activities. In addition, an early failure of the deeper current meter was detected, which, in a more critical setting, might have prompted an early mooring recovery and instrument replacement.

\section{a. Description of the buoy and base stations}

The basic elements of the buoy and base station telemetry equipment are shown in block form in Fig. 4. A primary objective was to minimize cost, so inexpensive amateur radio VHF transmitter/receivers and antennas were used for both stations. These units are readily available for use at frequencies near 145 , 220 and $430 \mathrm{MHz}$, and they are easily adaptable for the intended application. The pilot experiment telemetry frequency was $148.300 \mathrm{MHz}$, chosen for reasons of compatibility with the amateur equipment and for non-interference with existing services. Licensing requirements are discussed in Section 5.

A toroidal surface float $1.3 \mathrm{~m}$ in outer diameter and $0.3 \mathrm{~m}$ deep was used to support a $3 \mathrm{~m}$ section of TV antenna tower passed through the center. The radio and other electronic components were housed in a watertight case which was attached to the tower section. Two rechargeable batteries (Gel-Cells) were mounted in boxes attached to the toroid. An Aanderaa hydrophone receiver was mounted in a bracket at the bottom of the tower and approximately $50 \mathrm{~kg}$ of lead ballast was attached to it, to add stability. A $3 \mathrm{~m}$ vertical whip antenna was attached to the top of the tower section. The base of the antenna was about 1.5 $m$ above the sea surface.

To minimize transmitter and receiver modifications, an audio-frequency-shift keying (AFSK) modulation scheme was chosen for data and control functions. By transmitting coded sequences of standard digital telephone multiple-frequency (DTMF) tones, the buoy transmitter and the hydrophone receiver were activated for various predetermined time intervals. For the Aanderaa current meter application, $50 \mathrm{~s}$ intervals were used, and the basestation computer adjusted the turn-on time so that the $\sim 23 \mathrm{~s}$ Aanderaa data sequence occurred near the center of each transmission cycle. The Aanderaa data format includes a synchronization pulse, but it unfortunately occurs at the end of each record, so it is not useful as an automatic start pulse. More modern instruments with standard RS-232 data formats will be self-synchronizing. The Aanderaa pulse-width code was acoustically coupled to the surface buoy, where it was then used to key an AFSK modulator. Telephone AFSK tones were used (mark $=2295 \mathrm{~Hz}$, space $=2025 \mathrm{~Hz}$ ) but, for reasons described later, future versions will use standard radio-teletype AFSK frequencies.

Because of the weak-signal application, the receiving system used high-gain antennas and a low-noise preamplifier. At VHF frequencies, antenna gains of 18-20 dB relative to a dipole are obtainable with reasonable physical dimensions. The experiment used two vertically polarized, 14-element Yagi receiving antennas, separated $3 \mathrm{~m}$ and operated in-phase about $10 \mathrm{~m}$ above sea level. For refractive-mode propagation in a marine boundary layer, extreme antenna heights are not necessary and may be undesirable. A galeniumarsenide FET preamplifier with $24 \mathrm{~dB}$ gain and 0.5 $\mathrm{dB}$ noise figure was used during receive functions.

The received data consisted of an AFSK tone facsimile of the Aanderaa pulse code. The AFSK tones were decoded using a standard telephone MODEM (modulator-demodulator) circuit, which transferred the data to the serial interface of a HewlettPackard HP-87 computer. Because the Aanderaa code is non-standard, it was necessary to use one of 


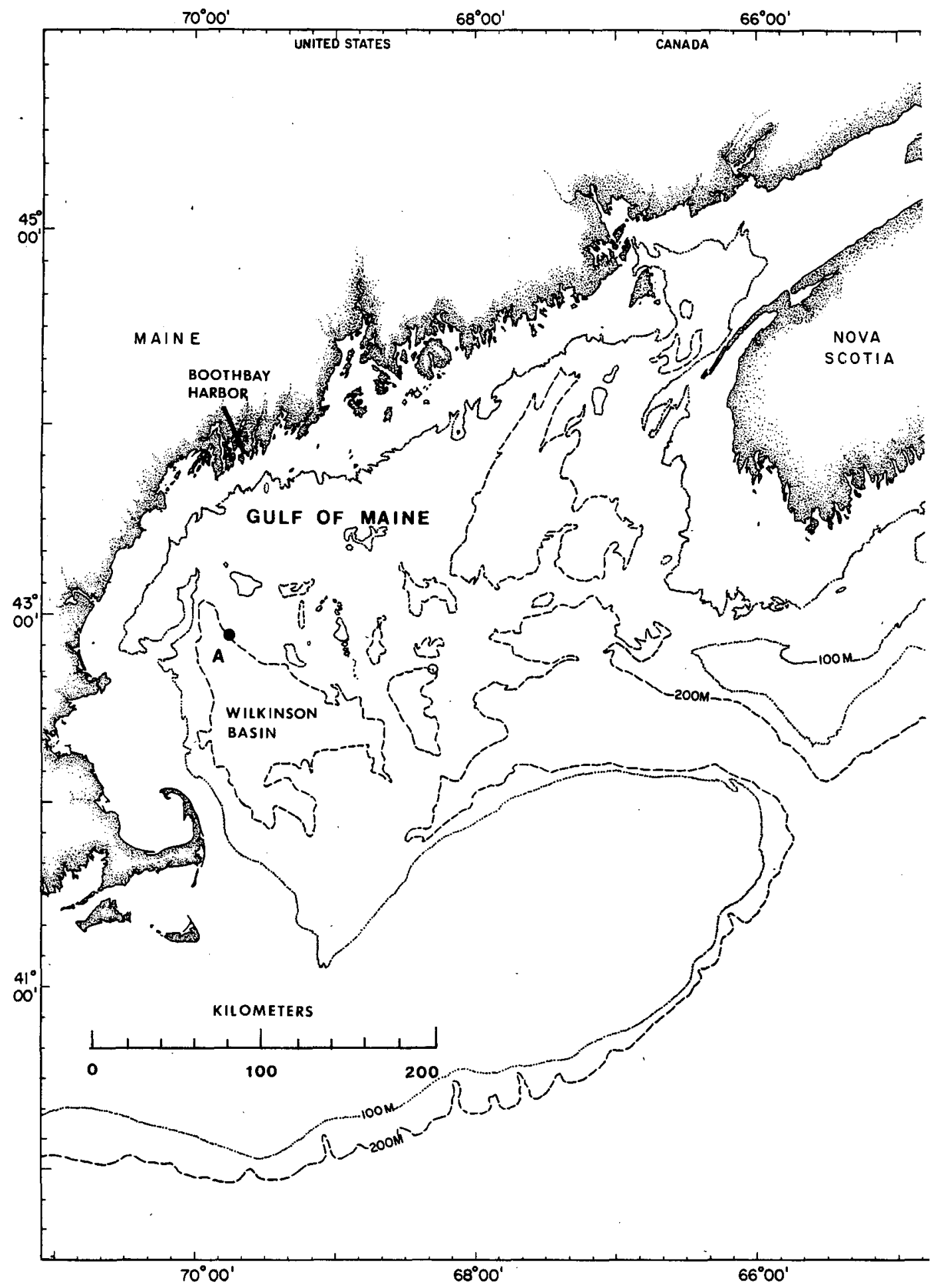

FIG. 3. Map of the Gulf of Maine, showing the location of mooring A during the 1983 field experiment. Mooring A was located $\sim 100 \mathrm{~km}$ south of Boothbay Harbor.

the RS-232 control lines to accomplish the data demodulation under software control by the computer, but future versions utilizing more modern instruments will be directly compatible with RS-232 data transfers.

\section{b. Telemetry system performance}

Mooring A was deployed on 2 June 1983. The surface telemetry buoy was tested on deck and in the 


\section{A. TELEMETRY BUOY}

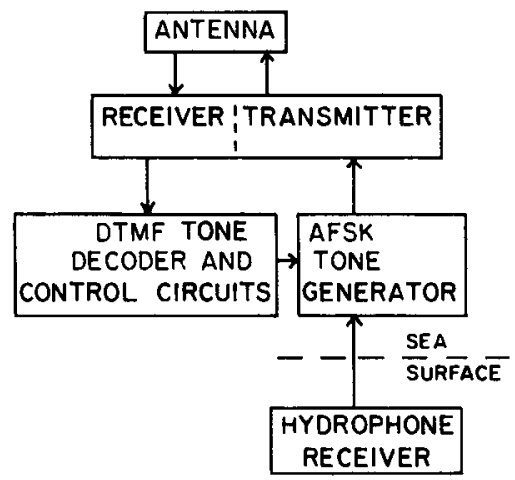

FiG. 4a. Block diagram of the electronic apparatus of the telemetry buoy.

water before the anchor was launched. After the mooring was in place, the top and bottom current meters were monitored for proper operation by sending the turn-on code from the ship. The current meter sampling intervals were pre-set at $20 \mathrm{~min}$, with a 10 min offset between instruments to avoid simultaneous transmissions. Both instruments were operating properly and several data records were recorded by the computer before the ship left the mooring area.

Very strong signal reception continued until the ship was about $10 \mathrm{~km}$ away from the mooring (about the line-of-sight distance). Beyond $10 \mathrm{~km}$, the signal strength dropped rapidly, and it soon became apparent that the telephone MODEM was a poor choice for a demodulator because of its high signal-to-noise ratio requirements. Future designs will use standard radioteletype demodulators, which are specifically designed for weak-signal applications. Because of the MODEM limitations, only a few data records were fully recorded by the computer, even though the signal strength often was sufficiently strong to allow direct, audible decoding of the Aanderaa binary pulse sequences.

Approximately two weeks later, the ship revisited the mooring site during the last transect of the cruise. The telemetry system was found to be working properly, but only the upper instrument could be heard. It was verified after the mooring was recovered in August 1983 that the battery in the lower instrument had failed a few days after deployment. During the intervening cruise operations, the most distant reception from the buoy was from $\sim 100 \mathrm{~km}$. The ship's telemetry antenna was a three-element Yagi which could be rotated manually. The receiver preamplifier was not available during the cruise.

After returning from the cruise, it was found that the telemetry control function of the computer at the base station was successful. Many partial data records were received, but the lack of demodulator sensitivity resulted in excessive bit errors. With practice, however, it became possible to read the Aanderaa data words audibly; that is, by manually noting the long and short pulses for each of the six 10-bit words. The binary words could then be converted to their decimalequivalents and then into scientific units using the appropriate calibration functions. The bit timing of the Aanderaa code is independent of the pulse width, which distinguishes it from a Morse-type code, but the skill required for audible decoding of the Aanderaa words is similar to that required for reading Morse code at 20-25 words per minute.

About one hundred records from the upper current meter were manually recorded during the mooring period, which ended 7 August. An additional number of signal strength determinations were recorded at various times by turning on the buoy transmitter for 10 second periods. Strength determinations were made subjectively in several classes, and objectively when the received signal was strong enough to give an indication on the receiver's digital strength indicator (S-meter). Both scales are logarithmic in nature, the former because of the sensitivity of the human ear, and the latter because of the S-meter calibration.

Examples showing the signal strength variations during the passage of three weather disturbances are given in Fig. 5. The first two occurred before the preamplifier was available; the detection level (minimum detectable signal strength) was significantly lower with the preamplifier during the third example. The strength scale is broken in its midsection because the dynamic range of the receiver was not known. While there is a considerable degree of subjectivity in signal-strength judgment (to be discussed shortly),

\section{B. BASE STATION}

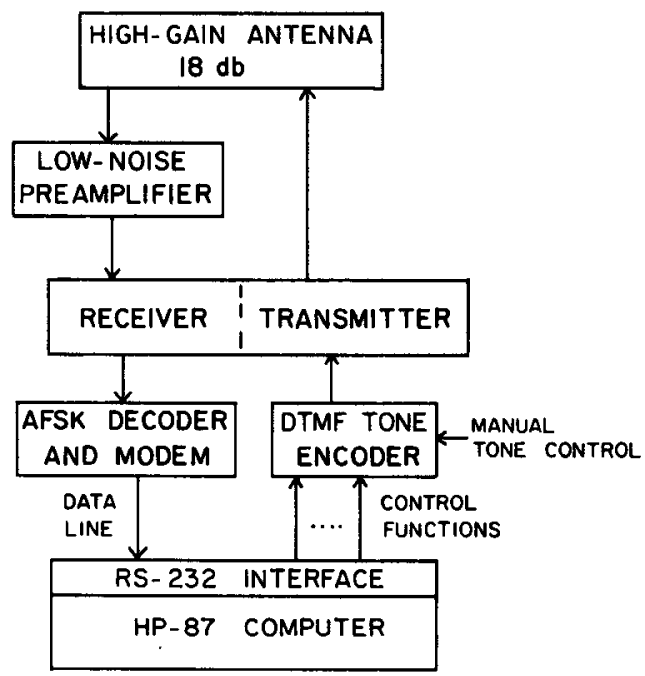

FiG. 4b. Block diagram of the base station electronics and computer interface. 


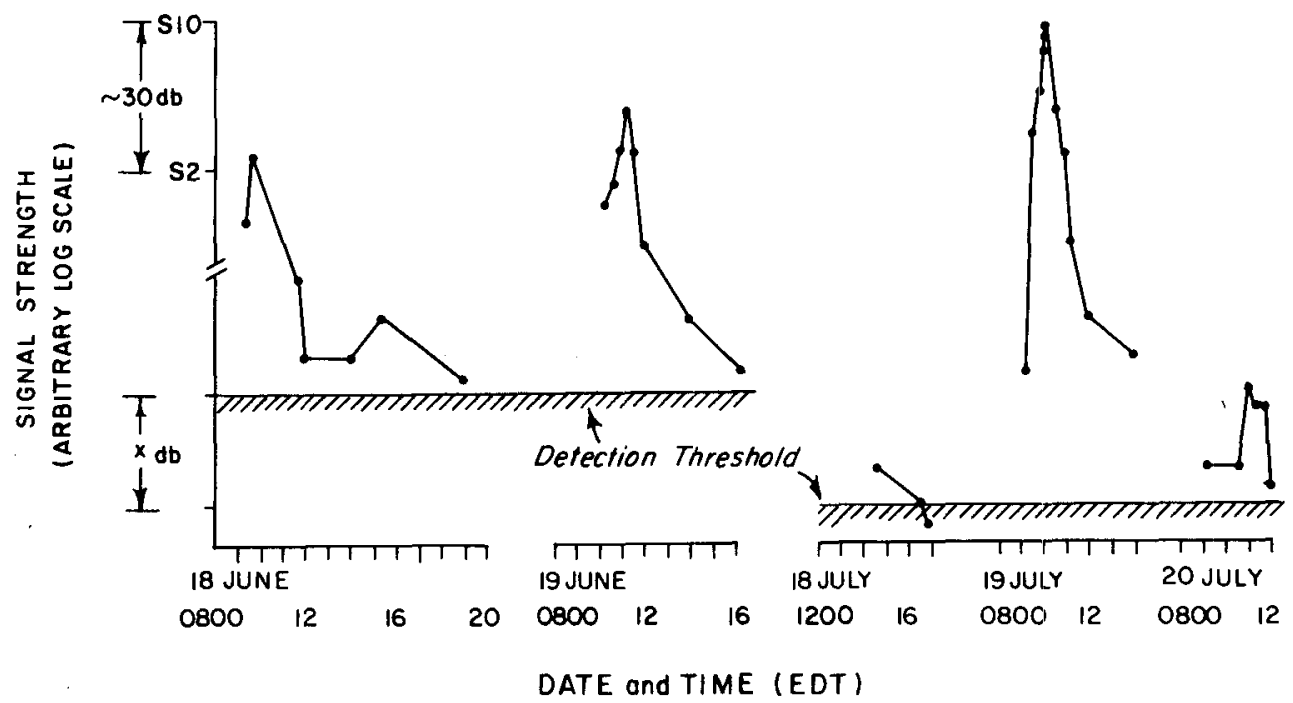

FIG. 5. Received signal strength variations during the passage of three weather disturbances. The arbitrary log scale is broken because the total dynamic range of the receiver was unknown. The detection threshold was lowered an undetermined amount $X$ (in the range 3-10 dB) when the preamplifier was used. During strong events, the preamplifier increased the $S$ meter reading on the Santec KDK-2030 receiver by $3-4$ units.

the extraordinarily large signal intensification during several frontal passages and the rapidity with which the changes occurred are obvious in the recorded data. The peaks which occurred on 18 and 19 June would have produced S-10 strengths if the preamplifier had been installed at that time. This is many orders of magnitude stronger than that required for nearperfect code detection, which level lies only slightly above the signal detection threshold.

All of the anomalously strong signal events in Fig. 5 were associated with the passage of atmospheric fronts over the Gulf of Maine. Daily and sub-daily satellite products from the NOAA Polar Orbiting and GOES Stationary Satellites were available at the basestation location, so the progression of weather events could be traced in sequences of infrared and visual images. Forenoon GOES images were usually available at 30 minute intervals.

The rapid signal strength increase between 1000 and 1100 local time on 19 June occurred just after a strong cold front moved offshore of the Maine Coast. A clear distinction between the warm, cloudy air offshore of the front and the colder, clear air behind it is evident in the NOAA infrared image shown in Fig. 6. The mottled gray areas with ragged edges are cold cloud tops. This image was taken at 0851 local time, about two hours before the signal strength reached its maximum. The local enhancement lasted only a few hours, until the frontal region moved seaward of the mooring location.

For orientation, the warm waters of the meandering Gulf Stream can be seen in the lower part of Fig. 6, and the seaward edge of Nova Scotia is also visible in the right, central part of the image. Boothbay Harbor, Maine lies under the frontal line in the area where it turns northward, and an extensive region of fog can be seen northwest of the front, over southern Maine. Several of the Great Lakes and part of the Gulf of St. Lawrence are evident north and west of the front, in the clear sector.

Logbook entries of signal strength were classified according to a quasi-subjective scheme which divided the full range of strengths into six categories, ranging from No Contact $(F)$ to Full Quieting (A). Intermediate categories were based on an audible judgment of noise "quieting," a peculiarity of frequency-modulation systems which is useful for signal-strength estimations. As FM signal strength increases, the noise audibility slowly decreases, until at some threshold level strong noise suppression occurs for only a moderate further increase in signal strength. This transition or "capture" point is quite noticeable, and it was chosen as the mid-point of the classification scale.

Frequency-of-occurrence and cumulative-occurrence graphs of the observed signal-strength classes are shown in Fig. 7. The graphs are based on 92 samples obtained from 11 June-21 July 1983 . Signal detection was possible about $75 \%$ of the time during which attempts were made to interrogate the buoy. Successful decoding occurred with slightly less frequency, probably near the $70 \%$ level. The most frequently observed signal-strength class was the one just below the point at which rapid receiver quieting 


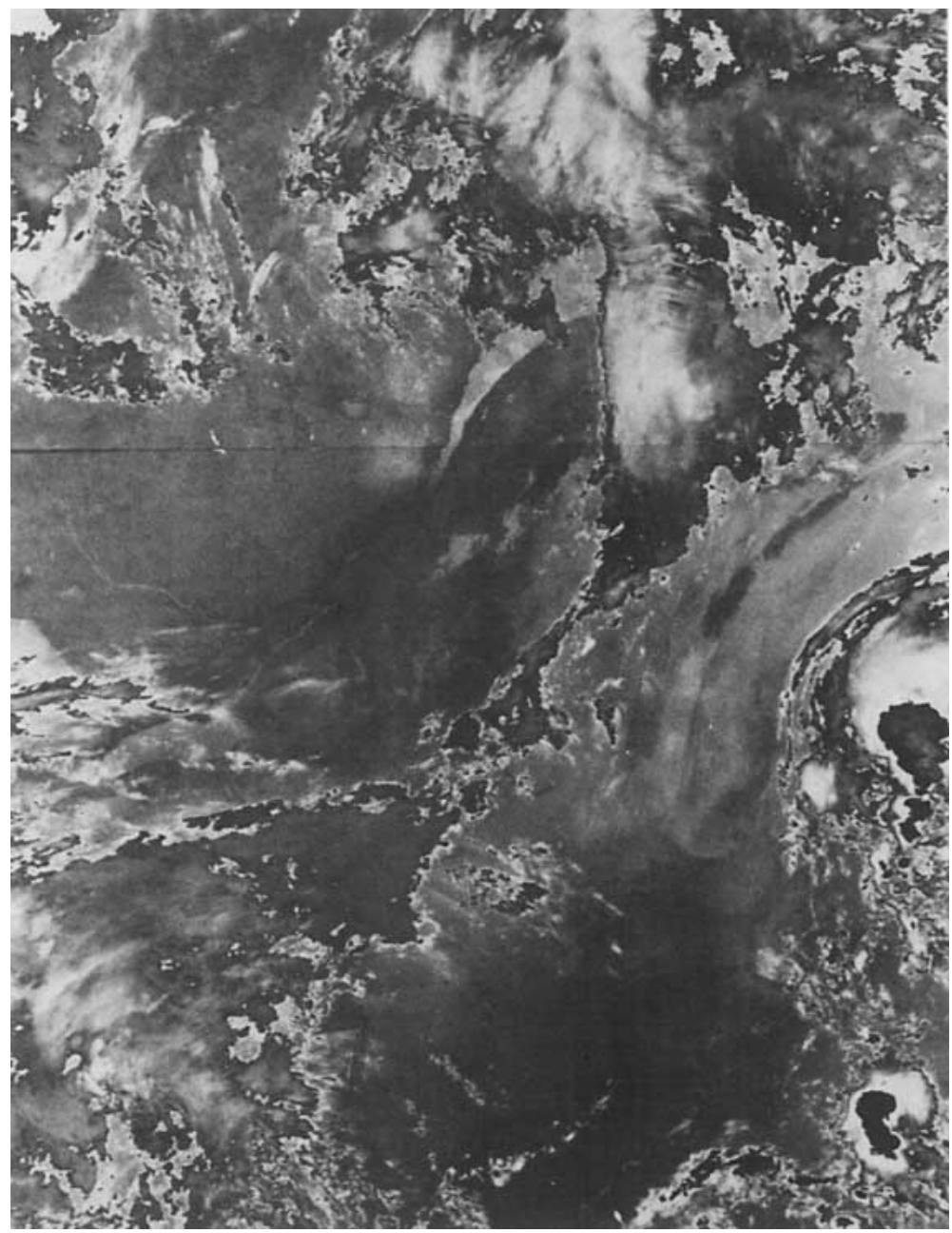

FIG. 6. Satellite infrared image taken a few hours before the signal enhancement that occurred on 19 June. A cold front was just passing over the southern Maine coast at the time of the image.

was noted, i.e., class D ("weak, some noise quieting") observations accounted for $38 \%$ of the total attempted during the period. The class-D level is well above the minimum decoding level, easily within the demodulation range of radio-teletype circuitry since manual demodulation was easily accomplished at that level.

The signal-strength data are shown in a time series format in Fig. 8. Each dot represents a single determination, except for dots connected by solid lines with an arrowhead, which indicates many observations during that period. Dashed lines which connect dots indicate absent data on a given day. In addition to the short-term weather effects just noted, there appears to have been a longer period signal-strength variation. For example, an interval of extremely weak signals occurred from 20-22 June and again from 12-14 July. During the latter period, the signal strength was below the detection level of the receiver without using the preamplifier. There is also a suggestion of diurnal modulation of the signal strength, probably caused by temperature inversions resulting from nocturnal radiative cooling of the atmosphere.

\section{An example of telemetered data}

In the mooring A region, historical data indicate that the non-tidal circulation consists of a slow, southeastward drift (Bigelow, 1927). Tidal currents are quite strong in the Gulf, and they may mask the tidal-residual pattern at times.

Figure 9 shows a vector diagram of the current speeds and directions telemetered from the $30 \mathrm{~m}$ depth on mooring A. Just the vector tips (dots) are shown for clarity. The observations were recorded manually at random sample times between 0800 and 2400 local time, so the semi-daily $\left(\mathrm{M}_{2}\right)$ tide is not well resolved in most of the daily sequences. However, the observations span a several-month period, during 


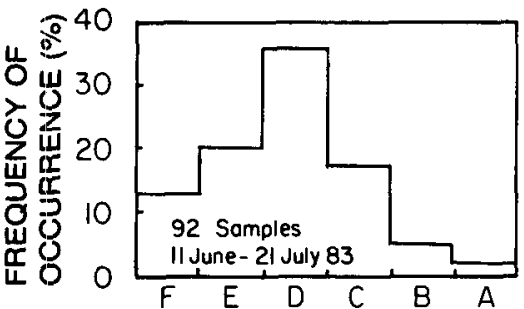

(W) SIGNAL STRENGTH CLASS (S)

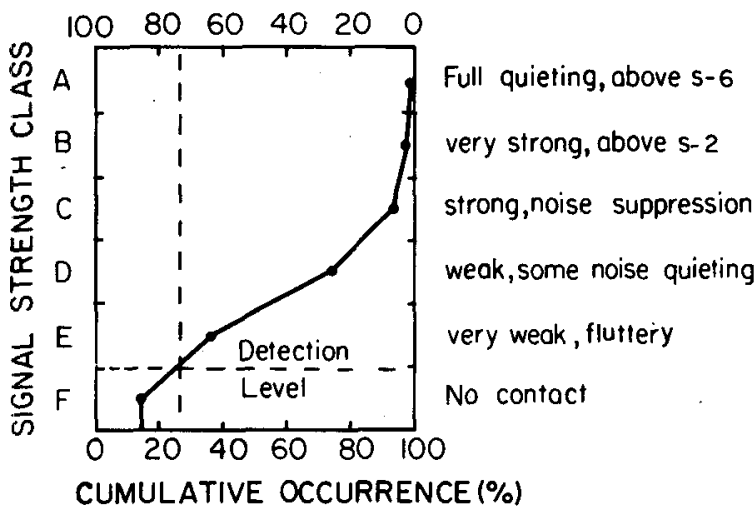

FIG. 7. Frequency distributions of the signal-strength classes received at the base station. Successful data transfer occurred on about $70 \%$ of the attempts.

which time the phase of the $\mathbf{M}_{2}$ tide twice shifted in and out of synchronization with the clock, so the daytime samples were randomly scattered throughout the entire phase cycle of the tide. Most of the current vectors have a southward component. The vector mean of the individual vectors is about $10 \mathrm{~cm} \mathrm{~s}^{-1}$ directed south, or slightly east of south, which is generally consistent with Bigelow's concept. The points scatter along a NNE-SSW line, which defines the major axis of the $\mathbf{M}_{2}$ tidal ellipse at the mooring site. Some of the current speeds were unexpectedly large, reaching a maximum of $40 \mathrm{~cm} \mathrm{~s}^{-1}$ toward the southeast during an ebbing tide on 23 July.

Aanderaa current meters provide for internal storage of data on a magnetic tape. After recovering the instruments from mooring $A$, the current speed and direction information was extracted using standard computer procedures. The data from the top instrument are summarized in Fig. 10, which is a normalized polar histogram of displacement obtained by vectorially accumulating the 3609 recorded velocity records in $10^{\circ}$ sectors. For comparison, the telemetered velocity data are overlaid as dots on the histogram. The southward current component is dominant in both the recorded and telemetered data. Since the histogram shows time-integrated currents, it emphasizes the southward direction, but the angular distribution of displacement is consistent with the orientation and magnitude of the telemetered velocity vectors.

\section{Licensing}

The Federal Communications Commission requires that telemetry transmitters of the type discussed here

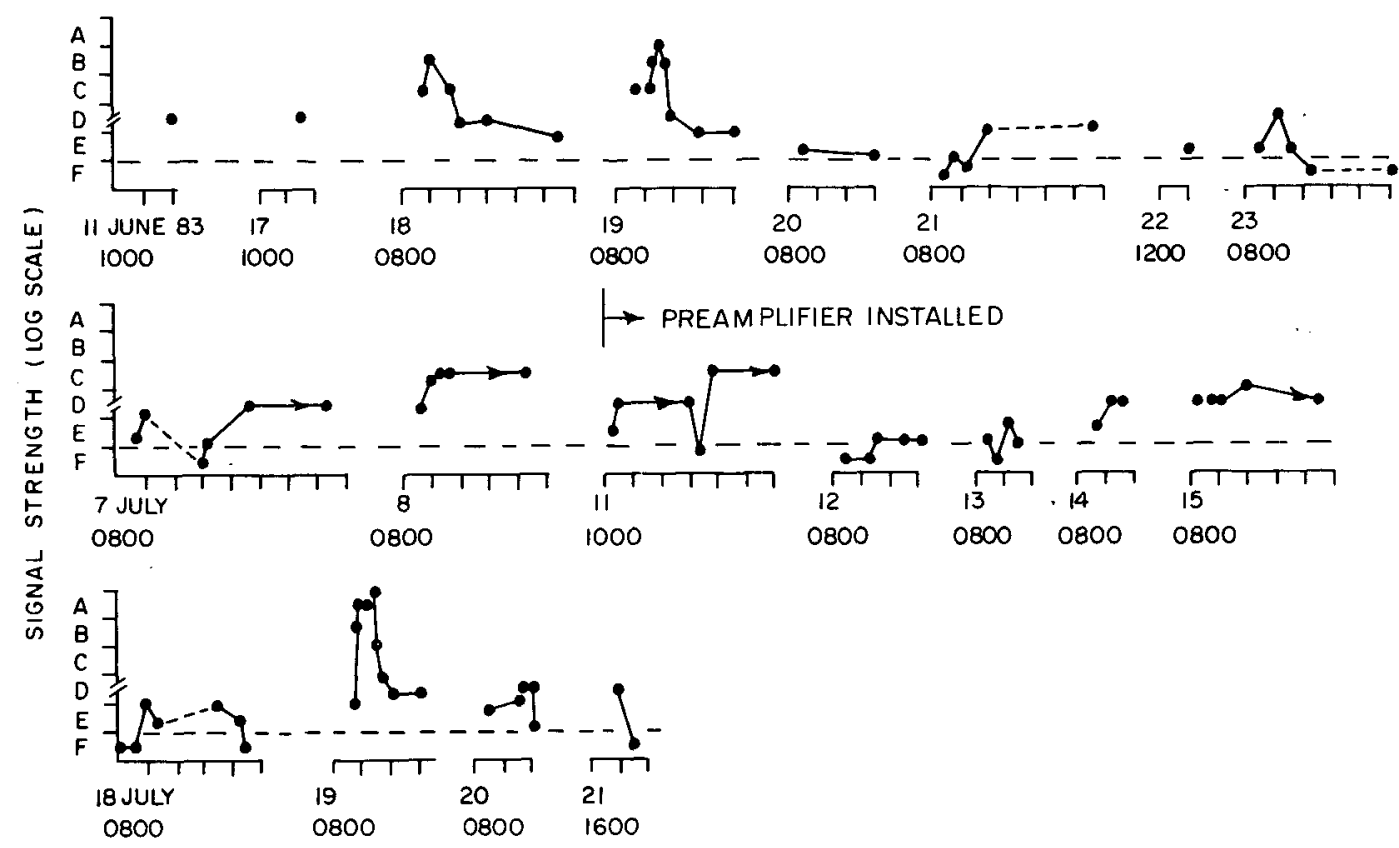

FIG. 8. Time-series format presentation of received signal-strength variations. The continuous dashed line is the detection level (minimum detectable signal strength). 


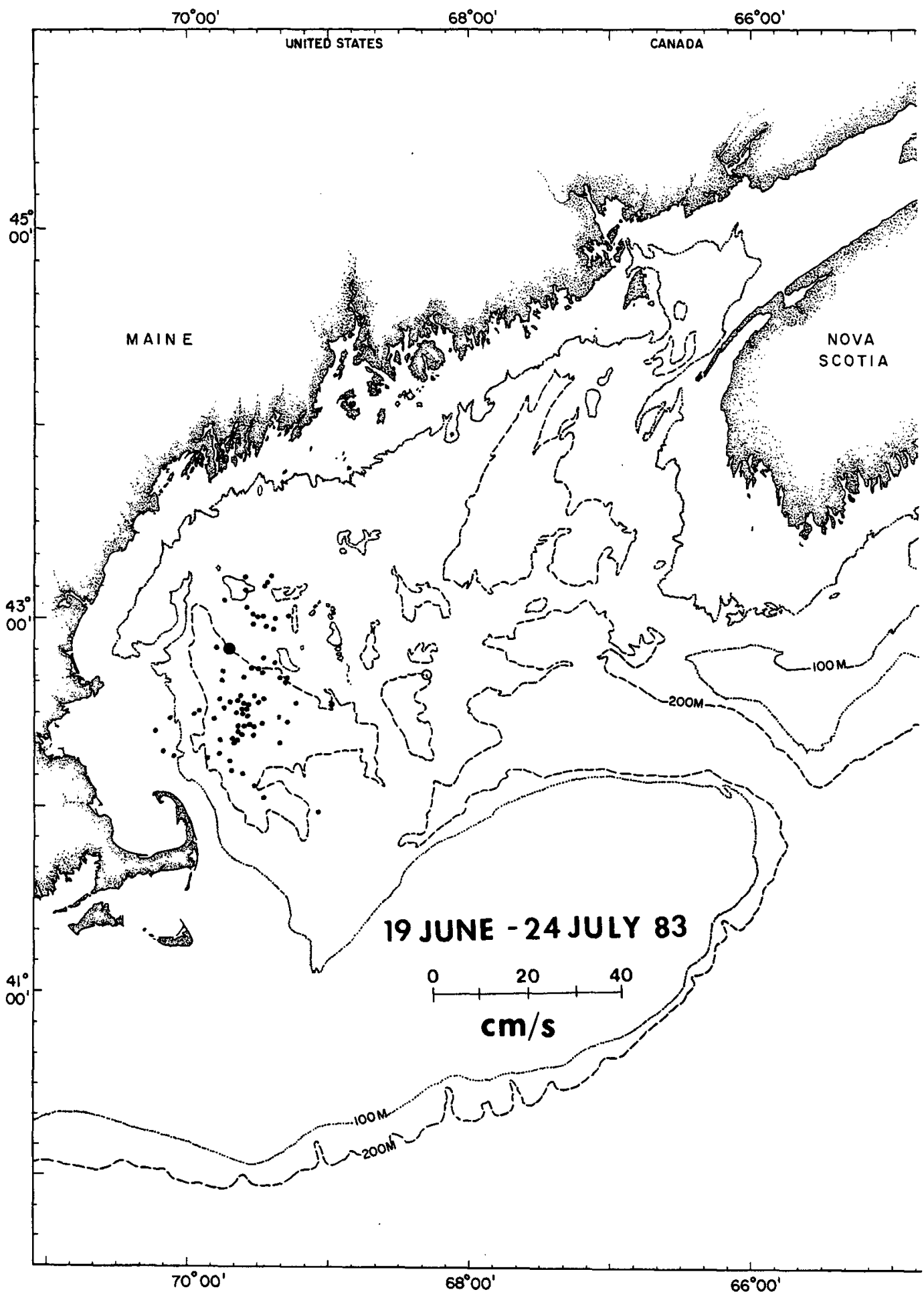

FIG. 9. A plot of velocity-vector tips, shown by the dots, relative to the mooring A position (large dot). These vectors were telemetered from the mooring and manually decoded, at random times between 19 June and 24 July 1983. The mean vector is about $10 \mathrm{~cm} \mathrm{~s}^{-1}$ toward the SSE, roughly consistent with the historical concept.

be fully licensed. The appropriate application is made on FCC form 442, which inquires about the particulars of equipment used, methods of insuring compliance with the Commission's rules, scientific justification, and assurance of non-interference with existing radio services. Practical VHF systems for transhorizon usage 


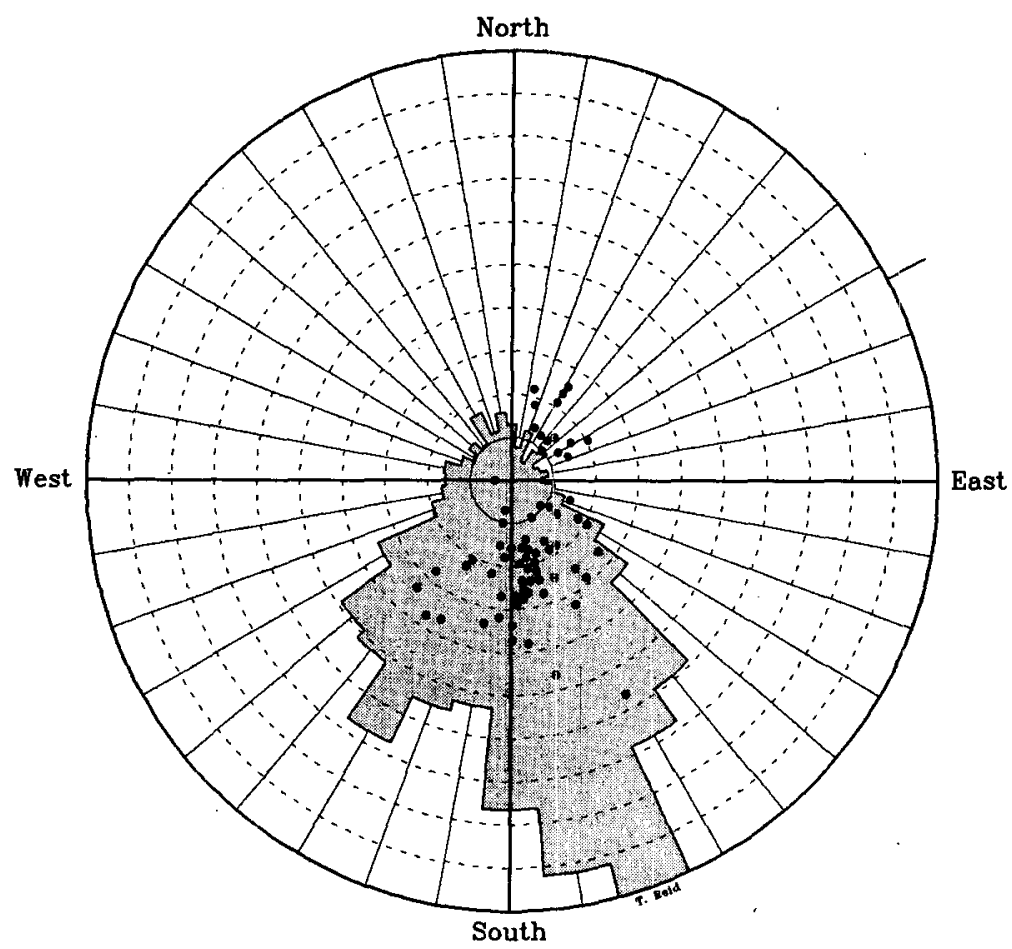

FIG. 10. Superposition of the telemetered current vectors (dots) and a polar histogram of current speeds vectorially-accumulated in $10^{\circ}$ sectors (shaded).

require buoy transmitter power levels in the range of 10-25 W, with antenna gains of 3-6 dB at the buoy and $15-20 \mathrm{~dB}$ or more at the receiver. Higher antenna gain is impractical on the buoy because its motion precludes horizontal directivity of the radiation pattern. Station licenses are issued for a specific location and for a specific time interval.

\section{Summary and conclusions}

A transhorizon VHF telemetry experiment was conducted as part of an ongoing study of the circulation in the Gulf of Maine. A mooring which supported Aanderaa current meters at depths of 30 and $180 \mathrm{~m}$ was equipped with a surface telemetry buoy which transmitted the instrument data to a shore station about $100 \mathrm{~km}$ distant. The combined height of the transmitting and receiving antennas was $12 \mathrm{~m}$ above sea level. The buoy and shore apparatus utilized inexpensive amateur radio equipment, which required minimal modifications. The telemetry system was operated from 2 June to 7 August 1983, during which time many current meter records were decoded and additional signal-strength determinations were made. It was found that successful data transfers could be made about $70 \%$ of the times attempted.

The received signal strength sometimes was greatly enhanced during synoptic weather events. On several occasions, line-of-sight strengths occurred and were maintained for $30 \mathrm{~min}$ or more as a cold front passed over the coastal area. Such enhancements evidently were associated with strong wave refraction in air layers supporting tropospheric temperature or moisture inversions. At most other times, in the absence of synoptic weather events, weaker but still useful signal strengths were noted, with propagation evidently maintained by refractive moisture gradients in the marine boundary layer.

Data transfers with a higher success level could be accomplished by providing a short-term capability for storing data on the telemetry buoy. With this resilience, data transfers could be made at high rates at times of favorable propagation, resulting in little loss of telemetered data at the cost of a mean delay of a few days. Greater transmission distances or lower buoy power levels than those reported here could probably be traded for longer data delays.

The close relationship between weather disturbances and VHF propagation enhancement provides a measure of predictability for data telemetry. With current satellite infrared and visible images, useful predictions of VHF "openings" can be made a day or two in advance. Detailed weather analyses, available on television cable weather channels, may also be useful for propagation predictions. Anomalous reception of TV programming is itself an indicator of imminent propagation enhancement.

There are many factors about refractive-mode telemetry remaining to be explored. For example, weaker atmospheric inversions in thinner layers will 
support ducting only at higher VHF or even UHF frequencies, so higher or multiple-frequency telemetry schemes might be advantageously considered. This process cannot be continued indefinitely because transmission path losses increase with increasing frequency, requiring higher power levels and antenna gains, and more accurate antenna aiming; on the other hand, antennas become smaller as frequency increases, and higher gains are more easily attained. As an indicator of the possibilities, refractive-mode communication has been established many times between California and Hawaii at frequencies near 144 and $220 \mathrm{MHz}$ and more recently between Italy and Spain at $1296 \mathrm{MHz}$.

Acknowledgments. The Gulf of Maine physical studies program and the experimental telemetry application were supported by the National Science Foundation under Grant OCE81-21971. The control circuitry for the telemetry buoy was designed and constructed at Texas A\&M by Mr. R. V. Pittman, who also assisted with instrument preparation and deployment. I am grateful to my friends and colleagues at the Bigelow Laboratory for Ocean Sciences, West Boothbay Harbor, Maine for office space, cruise assistance, and for tolerating my antennas in their beautiful field of view.

\section{APPENDIX}

\section{Characteristics of Weak-Signal Telemetry Systems}

The telemetry system discussed in this paper was designed to use inexpensive, commercially-available transmitters and receivers which are marketed primarily for amateur and marine radio applications. These units typically utilize "channelized" narrowband FM (NBFM) techniques with $-6 \mathrm{~dB}$ receiver bandwidths of $6 \mathrm{KHz}$, the primary advantage of NBFM over other methods being the noise quieting or "threshold" effect mentioned earlier. It is not clear, however, whether NBFM provides optimum performance in a weak-signal environment, where the most frequent realm of operation is near the threshold level. To address this issue, we briefly review some aspects of NBFM demodulators (limiter-discriminators) and channel information theory, in light of which the performance of the present telemetry system can be evaluated and suggestions for improvement offered.

\section{a. Signal detection in NBFM systems}

Mainly for spectrum economy, the carrier-frequency deviation in NBFM is limited to approximately the highest significant modulating frequency. For voice communications, this is typically $\sim \pm 3$ $\mathrm{KHz}$, permitting practical channel spacing as close as $\sim 10 \mathrm{KHz}$. The function of the demodulator is to extract a facsimile of the originally-encoded infor- mation, which of course is contaminated in transmission by noise and therefore degraded at the input of the demodulator. It is convenient to assess the demodulator's performance, i.e., its fidelity in extracting the original signal, in terms of signal-to-noise power ratios at the input and at the output.

Because the encoded information is carried in the instantaneous frequency (or phase), an FM discriminator is preceeded by a limiter, which suppresses undesired amplitude variations of the received signal. The desired output of the limiter is a phasor with constant amplitude but time-varying phase. The addition of a noise phasor produces random variation of the resultant phase. The threshold effect is readily demonstrated by calculating the mean-square phase variation as a function of input signal-to-noise ratio. The result shows a rapid decrease of phase noise in the output for a signal-to-noise ratio exceeding unity (e.g., Stremler, 1982, Chap. 6). When the signal strength falls below the noise threshold, the noise phasor is sufficiently large to cause abrupt phase changes in the output, by sweeping the resultant vector around its origin; this produces noise "spikes" in the output of the discriminator, which is sensitive to the time-rate-of-change of the phase.

The key elements, then, in the performance of a weak-signal NBFM receiver are the joint effects of limiting and threshold levels on the signal-to-noise ratio $S_{0} / N_{0}$ of the demodulator output. Middleton (1960, Ch. 15) shows that, for no limiting, $S_{0} / N_{0}$ $\propto a_{0}^{2}$ for $a_{0} \ll 1$ and $S_{0} / N_{0} \propto a_{0}$ for $a_{0} \gg 1$, where $a_{0}$ is the signal-to-noise ratio at the limiter input. The strong suppression of the signal is evident below the threshold $a_{0} \approx 1$. Similar results follow for the opposite extreme of "heavy" limiting, but, below the threshold level, limiting degrades $S_{0} / N_{0}$ because of signal-noise products produced in the nonlinear limiting operation [see Fig. A1, adapted from Middleton (1960)]. The important conclusion for weak-signal applications is that the voltage-limiting level should be set higher than the root-mean-square noise power, corresponding to $r_{0}>1$ in Fig. A1, thereby avoiding a $10-20 \mathrm{~dB}$ penalty in $S_{0} / N_{0}$ below the threshold level. Most commercial NBFM equipment is intended for strong-signal use, in which case additional limiting is not detrimental to signal output and may improve noise suppression, so some relaxation of limiter settings may be desirable when using such equipment for weak-signal telemetry. It is also evident in Fig. Al that NBFM systems offer no $S_{0} / N_{0}$ advantage over simpler amplitude-modulation (AM) systems, even when limiting is minimal in the former. Thus, NBFM might not be the best choice for a weak-signal system if equipment availability is not a factor.

\section{b. Channel-capacity, bandwidth and error-rates}

All information-transfer systems are subject to compromises between data-transfer rates, system 


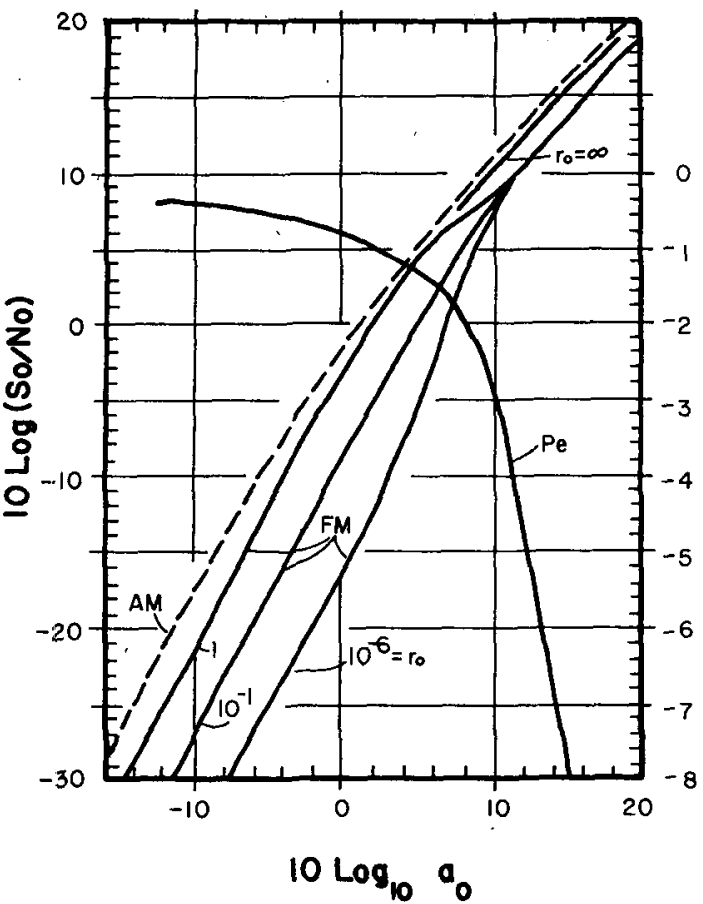

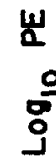

FIG. Al. Output signal-to-noise ratios $\left(S_{0} / N_{0}\right)$ of a demodulator for narrow-band frequency modulation (FM) as a function of input signal-to-noise ratio, $a_{0}$, shown for cases ranging from heavy limiting $\left(r=10^{-6}\right)$ to no limiting $\left(r_{0}=\infty\right)$ [redrawn from Middleton (1960)]. The dashed line applies to amplitude modulation (AM). Also shown is the probability of single-bit error $\left(P_{e}\right)$, as a function of $a_{0}$ [redrawn from Stremler (1982)].

bandwidth, and an acceptable probability of errors. A fundamental theorem of digital communication, due to Shannon (see Stremler, 1982), is that the datarate capacity of a band-limited channel containing Gaussian white noise is given by $C=B \ln (1+S / N)$ bits per second, where $B$ is the channel bandwidth, $S / N$ is the channel signal-to-noise ratio, and $C$ is the channel capacity. An optimum system is one in which the actual data transmission rate $R$ matches the channel capacity. For example, an optimum system with $B=6 \mathrm{KHz}$ and $S / N=10$ would have a maximum data-transmission rate of $\sim 14 \times 10^{3}$ bits $\mathrm{s}^{-1}$, which is about $10^{3}$ times the bit rate of Aanderaa current meters. Under-utilization of the channel capacity degrades $S / N$, because the receiver must contend with the noise in the unused bandwidth -On the other hand, the signal-to-noise ratio of the output of an ideal (noise-free) demodulator increases exponentially with the channel bandwidth for an optimum system (Stremler, 1982), so it is very desirable to use data-rates high enough to match the channel capacity.

In a digital pulse-code modulation (PCM) system, the $S / N$ of the quantized signal is $\sim n^{2}$, where $n$ is the number of quantization levels used, and all levels are assumed equiprobable. Thus, a 10-bit PCM system (e.g., Aanderaa), which resolves $n=1024$ levels, has a quantized $S / N \approx 60 \mathrm{~dB}$. For a sufficiently noisefree channel, the output $S_{0} / N_{0}$ of an ideal demodulator will therefore reach $\sim 60 \mathrm{~dB}$. For noisier channels or weaker signals, the output is degraded by thermal transmission noise, which is inversely dependent on the number of quantization levels. For a simplified case in which the demodulator details are not considered, Stremler (1982) shows that the output $S_{0} / N_{0}$ degrades to about $20 \mathrm{~dB}$ at the threshold level. However, Middleton's more detailed analysis shows that the best threshold performance which can be expected with $r_{0} \approx 1$ is $S_{0} / N_{0} \approx 8 \mathrm{~dB}$ (Fig. A1).

For a binary PCM system, the probability of error in bit decoding is related to $a_{0}$ as $P_{e}=\operatorname{erfc}\left(a_{0}\right)^{1 / 2}$, where erfc is the complementary error function (Stremler, 1982). For $a_{0}$ values near the threshold, $P_{e}$ $\approx 10^{-2}$, and the probability of more than one error in a 10-bit data word is about $5 \times 10^{-3}$. A graph of $P_{e}$ as a function of $a_{0}$ is included in Fig. A1.

\section{c. Recommendations}

The present telemetry system underutilizes the available $6 \mathrm{KHz}$ bandwidth, unnecessarily degrading the signal-to-noise ratio at the receiver input. The data transmission rate of an Aanderaa current meter is about 10 bits s $^{-1}$, which requires a bandwidth of only $10 \mathrm{~Hz}$ for equal signal and noise powers. The AFSK modulation adds a bandwidth requirement of about $4 \mathrm{KHz}$, so the data-transmission rate could be increased to about $2 \times 10^{3}$ bits s$^{-1}$ without exceeding the channel capacity. Alternatively, narrow filters could be used in the receiver, but a better plan is to transmit the data at much higher rates at times of favorable propagation conditions. However, the latter method requires temporary data storage at the transmitter, increasing the system complexity and cost.

Thermal (random) noise in the first amplifier stage of the receiver is one of the limiting factors in a weak-signal telemetry system. For this reason, it is advisable to use a high-gain, low-noise preamplifier, preferably located at the antenna terminals to avoid feedline deterioration of the overall receiver noise figure (e.g., Stremler, 1982).

Radiation from space produces an unavoidable random antenna noise called "sky-noise," which may be larger than preamplifier noise in the frequency range 100-200 MHz. The problem may be minimized by using high-gain antennas aimed away from noisy parts of the horizon, but the orientation of telemetry antennas will probably be determined by other factors. The sky-noise reaches a minimum in the $2-8 \mathrm{GHz}$ range, so a frequency higher than that used here (148 $\mathrm{MHz}$ ) might be advantageous for future systems. As noted earlier, ducting effects may also favor higher frequencies, which suggests that frequencies in the $\mathrm{GHz}$ (microwave) range might be considered for refractive-mode telemetry in cases where extreme antenna-pointing accuracy can be maintained (i.e., probably excluding ocean buoys). 
When using FM under weak-signal conditions, it is important that limiting not occur below the threshold signal-to-noise level $(\sim 8-10 \mathrm{~dB})$. Sub-threshold limiting can desensitize the receiver by $10-20 \mathrm{~dB}$, possibly negating the advantages of a low-noise preamplifier stage. Commercial receivers of the type used here are usually set to limit above the threshold level, but this setting should be checked and adjusted if necessary for optimum performance.

The bit-error rate for PCM decreases rapidly for signal strengths above the threshold level. To achieve the lowest possible error rate for given conditions of propagation, therefore, it is necessary to maximize the signal-to-noise ratio. This is accomplished, for example, by matching the data-rate to the channel, and by using a large number of digitizing levels to reduce quantization noise at the transmitter. Trans- mitted power can be increased, of course, but space and battery requirements usually limit power levels to a few tens of watts for telemetry applications; more beneficial improvements accrue from the use of lownoise receiver preamplifiers and remote antenna sites.

\section{REFERENCES}

Bean, B., and E. Dutton, 1968: Radio Meterology, Dover, 435 pp. Bigelow, H. B., 1927: Physical oceanography of the Gulf of Maine. Bull. Bur. Fish., 40, 511-1027.

Middleton, D. S., 1960: An Introduction to Statistical Communication Theory. McGraw-Hill, $1140 \mathrm{pp}$.

Stremler, F. G., 1982: Introduction to Communication Systems. Addison-Wesley, $702 \mathrm{pp}$.

Unwin, R. S., 1951: Report of factual data from the Canterbury Project, Vols. I-III. Dept. Sci. and Ind. Res., Wellington, New Zealand (for excerpts see Bean and Dutton, 1968). 